\title{
Development of an in-sole plantar pressure measurement device
}

\begin{abstract}
This paper describes the design and development of an In-sole Plantar Pressure Device (IPPD) to measure underfoot pressure. Knowledge on underfoot pressure is important for different purposes. It is essential for doctors and clinicians to have information about underfoot pressure to enable them to diagnose foot problems. Currently, underfoot pressure ranges have not been effectively established, and there is insufficient information about the minimum and maximum values of underfoot pressure. Likewise, constraints with regard to the implementation of insole systems still persist and are represented by the need to put a spacer on the sensor during measurements, and the problem of sensor parameters changing after the calibration. These challenges are addressed in this work and preliminary results or performance of the proposed device are then presented.
\end{abstract}

Keyword: Plantar pressure; Underfoot pressure; Insole device 